\title{
Mensuração da percepção estética de aparelhos auditivos por meio do rastreamento ocular
}

\section{Aesthetics measurement of hearing aids through eye tracking: a contribution of design for inclusion}

Julia Marina Cunha ${ }^{1}$

Giselle Schmidt Alves Díaz Merino ${ }^{2}$ 


\section{Resumo}

As características estéticas dos produtos influenciam nas interações que se tem com os mesmos, assim como podem alterar os significados atribuídos a eles. Em se tratando de tecnologias assistivas essas associações podem levar ao reforço do estigma de incapacidade com relação aos seus usuários. Assim, essa pesquisa teve como objetivo mensurar a experiência estética visual com 3 diferentes modelos de aparelhos auditivos, por meio do rastreamento ocular. Dessa forma, possibilitando identificar como os princípios de estética considerados se comportam em aparelhos auditivos, de acordo com os padrões visuais identificados. Além do rastreamento ocular, utilizou-se de questionário para obtenção de dados acerca dos sujeitos. Como principais resultados, identificouse maior preferência pelo modelo de aparelho com maior grau em novidade, assim como foi possível verificar maior concentração do olhar nos modelos de aparelho preferidos pelos sujeitos. Além disso, corrobora-se com a literatura ao identificar relações entre duração das fixações e proporção, assim como número de fixações e pureza. Entende-se que ao mensurar a experiência estética de modo objetivo, pode-se compreender aspectos cognitivos e afetivos da interação com aparelhos auditivos.

Palavras-chave: Estética, Tecnologia assistiva, Design, Rastreamento ocular Aparelho auditivo.

${ }^{1}$ Mestra - UFSC (juliamarinac@gmail.com)

2 Doutora - UFSC (gisellemerino@gmail.com)

\section{Introdução}

A formação das atitudes do ser humano é constantemente guiada pela estética visual dos estímulos com os quais interage, onde estética pode ser definida como a apreciação sensorial que se tem quando em contato com o estímulo (HEKKERT, 2014). Em se tratando de produtos, as características estéticas interferem diretamente na interação do usuário com o mesmo, uma vez que é por meio desta que se tem o primeiro contato com o produto (LOBACH, 2001). Por conseguinte, a experiência estética é um fator primário para o sucesso do mesmo no mercado, interferindo também nas relações de uso (PHAM, 1999). Embora a experiência estética, compreenda todos os sentidos, o sentido visual é predominante, sendo responsável pela maior quantidade de informações acerca do mesmo (HSIAO; CHIU; CHEN, 2008).

Além disso, a estética do objeto influencia também nos significados atribuídos a ele (PATRICK, 2016), especialmente em produtos de Tecnologias Assistivas (TA), - processo de significação social tende a associar o uso desses produtos com características depreciativas, como incapacidade ou fragilidade (GOFFMAN, 2018). Essa estigmatização interfere diretamente na qualidade de vida do usuário, prejudicando a autoestima e podendo levar ao abandono da tecnologia assistiva (PAPE; KIM; WEINER, 2002).

Nesse sentido, destaca-se a importância do fator estético para promoção da inclusão de Pessoas com Deficiência (PcD), nesse estudo especificamente, concentrase em explorar esses aspectos em aparelhos auditivos. Esses, são dispositivos fundamentais para a comunicação e independência dos usuários, no entanto mantêm-se no projeto dos aparelhos algumas características estéticas que reforçam os estereótipos da deficiência ao relacionarem-se à dispositivos médicos (JACOBSON, 2014; PULLIN, 2009). Entende-se que para projetar de modo a promover a inclusão da pessoa com deficiência, é necessário compreender as relações entre a estética do produto e a percepção que se tem do mesmo.

Para tanto, verifica-se a necessidade de utilização de métodos de mensuração da experiência estética. Sendo o rastreamento ocular, um método que permite a investigação da percepção do observador quanto a reação em tempo real aos produtos, vêm sendo utilizado para avaliar a experiência com produtos (GUO et al., 2016; HO; LU, 2014; KHALIGHY et al., 2015). A análise de padrões visuais por meio do rastreamento ocular, possibilita relacionar as características estéticas do produto com reações sensoriais ao mesmo, e assim explorar como tais características podem interferir na relação com a Tecnologia Assistiva.

Assim, essa pesquisa tem como objetivo mensurar a percepção estética visual de 3 diferentes modelos de aparelhos auditivos por meio do rastreamento ocular, determinando a preferência dos participantes em relação às características estéticas dos produtos. Deste modo possibilitando identificar como os princípios de estética considerados se comportam em aparelhos auditivos de acordo com os padrões visuais identificados.

\section{Fundamentação Teórica}

Neste capítulo são apresentadas as bases teóricas que fundamentaram o desenvolvimento desta pesquisa, discorre-se principalmente sobre os tópicos: Tecnologia assistivas, Estética e Rastreamento ocular. 


\subsection{O aparelho auditivo como Tecnologia Assistiva}

O aparelho auditivo, sendo uma TA de auxílio à comunicação, é utilizado para recuperar parcialmente a capacidade auditiva de indivíduos com deficiência promovendo, portanto, a inclusão. Contudo, verifica-se que por vezes a função estética acaba por ser negligenciada no projeto desses dispositivos, o que afeta diretamente os significados atribuídos por usuário e sociedade, ao produto (PULLIN, 2009).

Nesse sentido, de acordo com Pape, Kim e Weiner (2002), os produtos assistivos estão socialmente associados com características depreciativas, tais como desonra e constrangimento. O que impacta diretamente a identidade social e autoestima do usuário, uma vez que os produtos assistivos tendem a estar ligados ou próximos ao corpo e, portanto, ajudam a compor a aparência do indivíduo (SHINOHARA; WOBBROCK, 2011). Ao longo da existência humana, o corpo é suporte e meio de produção de linguagem (KATZ, 2008), e os objetos utilizados junto ao mesmo atuam como modificadores de significados. $O$ aparelho auditivo, como um desses objetos, mantém ainda hoje características estéticas relacionadas à tentativa de esconder ou camuflar o produto durante o uso, como a miniaturização ou o uso de cores de pele (PULLIN, 2009).

Portanto, a necessidade de gerar uma imagem positiva para esses produtos, visando a melhoria da autoestima e consequente qualidade de vida dos usuários Sendo 'estética' a ciência das aparências percebidas pelos sentidos (LOBACH, 2001), é aplicada ao processo de design como meio de significar o objeto.

\subsection{Estética visual}

Tem-se, por definição, a estética como experiência sensorial da qual deriva uma resposta imediata ao estímulo (HEKKERT, 2014). Enquanto a percepção humana governada pela visão, a percepção visual é guiada pela atratividade de um estímulo (NAYAK; KARMAKAR, 2019). Nesse sentido, a estética visual interfere na percepção sobre um indivíduo, e em se tratando de produtos modifica a relação funcional com os mesmos, produtos percebidos como mais bonitos tendem a ser percebidos também como mais funcionais, de fácil utilização (DIEFENBACH; HASSENZAHL, 2011).

Além de proporcionar prazer sensorial, a função estética dos produtos tem como objetivo comunicar uma informação, nesse sentido o projetista seria a fonte da mensagem; o produto, transmissor e os sentidos, receptores (CRILLY; MOULTRIE; CLARKSON, 2004). Devido a natureza plural e subjetiva da estética não é possível chegar a uma conclusão única e unânime para determinar as variáveis da estética visual, é também por isso que diversos estudos consideram variáveis diferentes (MOSHAGEN; THIELSCH, 2010; NAYAK; KARMAKAR, 2019). Portanto, tendo em vista o objetivo dessa pesquisa, considera-se a teoria de Khalighy et al. (2015), que relaciona princípios tangíveis de beleza e atração, com padrões visuais identificados por meio de rastreamento ocular para mensuração da estética.

Segundo Khalighy et al. (2014), os elementos que compõe a informação estética são divididos principalmente em beleza e atração. Enquanto beleza é um fenômeno constante, não afetada por estímulos externos, atração é variável e sofre interferência de fatores externos, como cultura ou experiências prévias do indivíduo (KHALIGHY et al., 2014). Beleza e atração correspondem respectivamente à agradabilidade e atratividade na percepção do produto (Figura 3).

Nessa perspectiva, a beleza consiste nos princípios de design formados com base na percepção do ser humano de características agradáveis (NORMAN, 2008), apresentando uma certa previsibilidade, é composta por princípios tangíveis:

- Contraste: pode ser definido como a diferença entre elementos que gera reconhecimento visual (COATES, 2003), essa diferença depende de quantos elementos são visíveis e quanto são diferentes entre eles.

- Proporção:é o equilíbrio de pesos entre os elementos, gerado pelas características visuais dos mesmos (KHALIGHY et al., 2015).

- Pureza: é o que define o nível de simplicidade ou complexidade de um estímulo, relacionado ao número de elementos de um estímulo visual (NORMAN, 2008).

Já atração é um fenômeno relativamente dependente de características individuais, portanto dependente de variáveis intangíveis, são elas:

- Familiaridade: Alguns estudos demonstram que o ser humano tende a ser atraído pelo o que é reconhecido, princípio este que possui raízes evolutivas, sendo mais seguro se aproximar do que é familiar (HEKKERT, 2014). Tratando-se de produtos, o ser humano tende a valorizar certo grau de tipicidade (BERGHMAN; HEKKERT, 2017).

- Novidade: Ao mesmo tempo, devido também aos processos evolutivos, o ser humano deriva prazer do processamento de objetos diferentes, sendo atraído por produtos percebidos como novos ou inovadores (HEKKERT, 2014).

Assim, os princípios de estética apresentados são relacionados com as reações fisiológicas visuais para possibilitar a sua mensuração (KHALIGHY et al, 2014), nos objetos de estudo selecionados, por meio do rastreamento ocular.

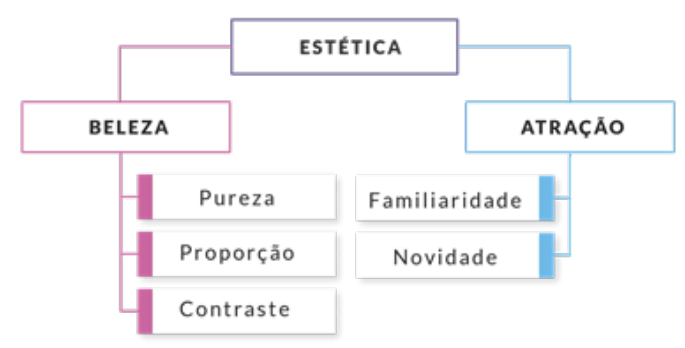

Figura 3- Diagrama dos princípios da estética segundo Khalighy et al. (2014)

\subsection{Rastreamento ocular}

As percepções do usuário com relação ao produto são, principalmente, afetadas pela aparência do produto. Além disso, grande parte das emoções provocadas por um produto são mediadas por uma percepção visual inicial (GUO et al., 2016). A informação visual corresponde a $80 \%$ das informações sensoriais obtidas durante uma experiência estética (DOUCHOVA; NESETRIL, 2009), por isso o rastreamento ocular vem sendo utilizado como uma técnica de avaliação da estética em produtos (NAYAK; KARMAKAR, 2019). Assim, o rastreamento ocular permite a mensuração das reações sensoriais oculares (GOBBI et al., 2017), podendo posteriormente serem interpretadas para compreender a experiência estética.

O eye-tracking ou equipamento de rastreamento ocular, fornece dados sobre o comportamento visual do usuário por meio do registro dos movimentos oculares. As 
métricas consideradas neste estudo, devido as relações possíveis para mensuração da estética (KHALIGHY et al.,2015), são:

- Fixação do olhar: Considera-se fixação quando o olhar permanece estável em uma área da exibição visual durante 200-300ms (GRANKA; FEUSNER; LORIGO, 2008). Podem ser analisados fatores como número de fixações e tempo que o participante concentrou a atenção visual em uma determinada área.

- Dilatação da pupila: É identificada por meio do aumento no diâmetro da pupila podendo indicar interesse ou atração por um produto (GUO et al., 2016).

\section{Procedimentos Metodológicos}

Neste capítulo, apresenta-se a caracterização geral da pesquisa, assim como as etapas em que foi subdividida, e os métodos adotados.

\subsection{Caracterização da pesquisa}

Essa pesquisa classifica-se como aplicada no que tange a sua natureza, uma vez que visa gerar conhecimento acerca de um problema específico. Do ponto de vista dos objetivos, pode ser classificada como exploratória, pois visa explorar informações acerca do assunto estudado, nesse caso a percepção estética de tecnologias assistivas (PROVDANOV; FREITAS, 2013).

Com base nos procedimentos técnicosé classificada como pesquisa experimental, onde selecionam-se as variáveis e um objeto de estudo, buscando investigar os efeitos das variáveis nos objetos (PROVDANOV; FREITAS, 2013).

\subsection{Etapas da pesquisa}

A presente pesquisa foi dividida em 3 etapas, a primeira de identificação da problemática, a segunda de coleta de dados, e a terceira de análise dos dados coletados (Figura 1).

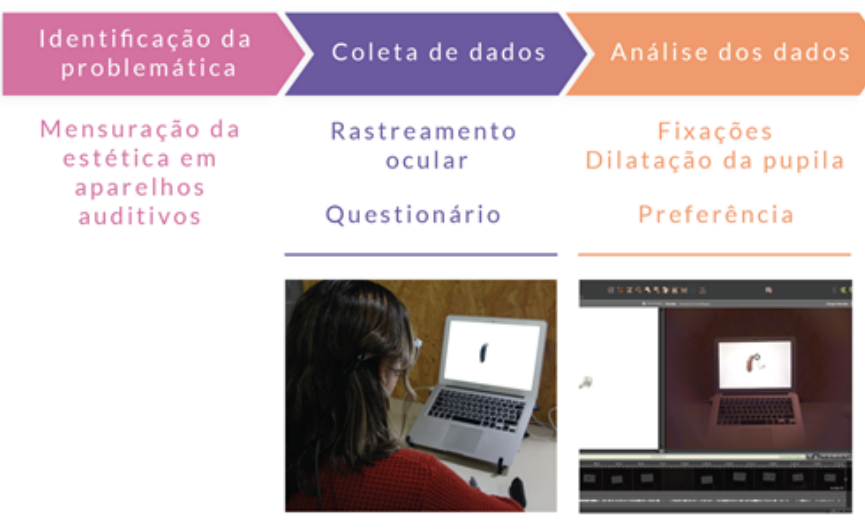

Figura 1- Etapas da pesquisa

\subsection{Materiais e métodos}

O experimento foi realizado com 29 sujeitos, indivíduos de ambos sexos, entre 20 e 55 anos que se dispuseram a participar do estudo com o uso do rastreamento ocular, todos os participantes não-usuários de aparelho auditivo, além disso não houve delimitação quanto à experiência prévia dos sujeitos. Ainda, destaca-se que não delimitaram-se como participantes os usuários de aparelho auditivo pois não foram observadas questões relativas ao uso, e sim quanto à percepção visual dos observadores com relação aos produtos.

A coleta de dados foi realizada em Bauru/SP durante o evento CBTA (Congresso Brasileiro de Pesquisa e Desenvolvimento em Tecnologia Assistiva). Antes de iniciar o experimento, os participantes tomaram conhecimento e fizeram a assinatura dos Termos de Consentimento Livre e Esclarecido (TCLE), e Uso de Imagem e Voz.

Quanto aos objetos de estudo, foram selecionados 3 modelos de aparelhos auditivos retroauriculares (Figura 2):

- Aparelho A: Modelo retroauricular de uso mais comum comercializado atualmente, em cor de pele.

- Aparelho B: Modelo de retroauricular comum, comercializado atualmente, na cor preta.

- Aparelho C: Proposta de aparelho auditivo com características estéticas de acessório de moda (CUNHA; MERINO, 2018), não comercializado, na cor branca.
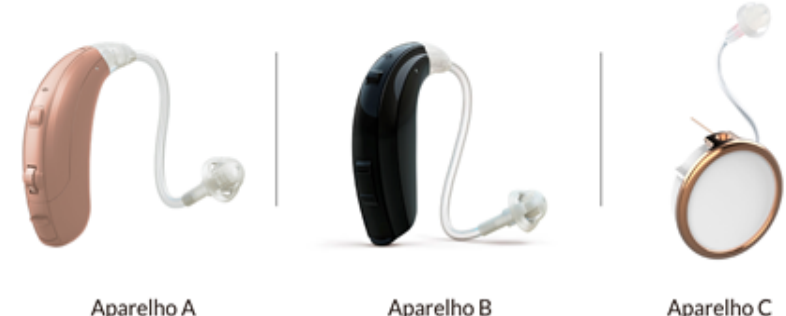

Figura 2- Modelos de aparelho auditivo apresentados no experimento

O modelo de Aparelho $C$ é um projeto conceitual que busca reduzir o estigma associado ao dispositivo, por meio da desconstrução das características estéticas usualmente aplicadas aos modelos de aparelho auditivo, associando-se a um acessório de moda (CUNHA; MERINO, 2018).

Assim, entende-se que os dispositivos selecionados apresentam diferentes características visuais, assim possibilitando identificar como os princípios de estética considerados se comportam de acordo com os padrões visuais identificados.

\subsection{Rastreamento ocular}

O equipamento de rastreamento ocular permite captar as reações fisiológicas visuais à um dado estímulo, possibilitando identificar os locais e duração das fixações visuais, assim como dilatação da pupila. Desse modo, viabilizando a quantificação das qualidades da estética. $O$ experimento foi conduzido utilizando o equipamento SMI-iView. Os participantes observaram as imagens em tela de computador 13" e o 
experimento foi acompanhado por meio do software iView. O procedimento envolveu 2 (dois) momentos:

1. Olhar livre: observação livre de imagens de três modelos de aparelhos auditivos apresentados individualmente na tela durante dez segundos (10s) cada.

2.Tarefa orientada: apresentação de dois modelos de aparelhos auditivos simultaneamente em cada tela, orientando para que o sujeito escolhesse o de sua preferência em termos de percepção estética, verbalizando a preferência em direita (aparelho da direita) ou esquerda (aparelho da esquerda). Foram apresentadas 3 telas com dois modelos de aparelho cada, assim exibindo todas as combinações possíveis.

Além da utilização do equipamento de rastreamento ocular, fez-se uso de um questionário aplicado ao fim do experimento para coletar informações sobre os sujeitos. A análise dos dados coletados com o equipamento foi feita com auxílio do software BeGaze 4.0, onde realizaram-se as marcações das áreas de interesse correspondentes a cada modelo de aparelho auditivo. Também por meio do software foi possível obter dados das fixações em cada área de interesse, assim como tempo das fixações e dilatação da pupila. Os dados obtidos do rastreamento ocular foram relacionados à princípios de estética assim como aos dados qualitativos do questionário. Após analisados, são apresentados os valores médios e desvio padrão do número de fixações nas áreas de interesse dos aparelhos A, B e C, assim como, valores médios do desvio padrão de duração das fixações.

Faz-se relação entre pureza (princípio estético) e número de fixações (dado do rastreamento ocular), proporção (princípio estético) e desvio padrão da duração das fixações (dado do rastreamento ocular). Sendo contraste uma relação entre ambos. Já a atração (princípio estético) é mensurada por meio dos valores de diâmetro da pupila (dado do rastreamento ocular), assim como percepções declaradas no questionário.

\subsection{Questionário}

Além de informações sobre o indivíduo, o questionário compreendeu também uma escala semântica para identificar a percepção do usuário quanto ao nível de inovação do produto utilizando os pares de adjetivo "familiar" e "inovador" com 7 níveis não numerados entre eles. A escolha dos adjetivos se deve as definições de estética apresentadas na fundamentação teórica referentes a familiaridade e novidade. Atribuíram-se valores de -3 a 3 , respectivamente aos extremos da escala para assim cálculo do índice.

1 O tipo de aparelho retroauricular é o mais difundido popularmente, é caracterizado pela estrutura da caixa de componentes ficar escondida atrás da orelha durante o uso (POPELKA et al., 2016).

\section{Resultados e Discussão}

São apresentados e discutidos os resultados obtidos do rastreamento ocular e questionário, relacionando-os com os princípios de estética considerados.

\subsection{Rastreamento ocular}

Considerando os resultados quantitativos do rastreamento ocular da tarefa de olhar livre, apresentam-se os dados por meio de gráficos, relacionando-os com os conceitos e demais dados demonstrados.

Assim, no gráfico a seguir (Figura 4) observa-se o número de fixações na área de interesse correspondente à cada modelo de aparelho auditivo. Como apresentado na fundamentação, pureza é relacionada ao número de elementos de uma composição, entende-se que o sujeito tende a fixar o olhar em todos os elementos identificados, sendo portanto, a pureza inversamente proporcional ao número de fixações em determinada área de interesse (KHALIGHY et al., 2015).

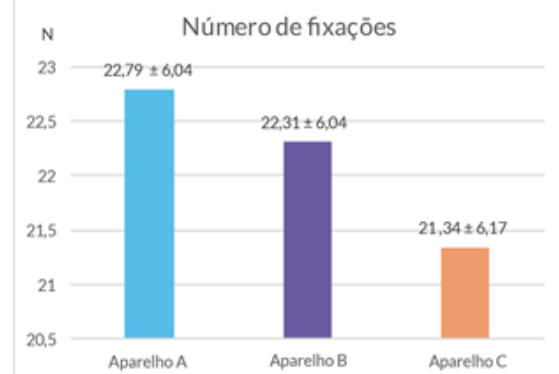

Figura 4- Gráfico número de fixações

Pela definição de proporção, tem-se que elementos proporcionais em uma mesma composição atraem o olhar pelo mesmo período de tempo, sendo proporção inversamente proporcional ao desvio padrão da duração das fixações em determinada área de interesse, dados apresentados no gráfico a seguir (Figura 5).

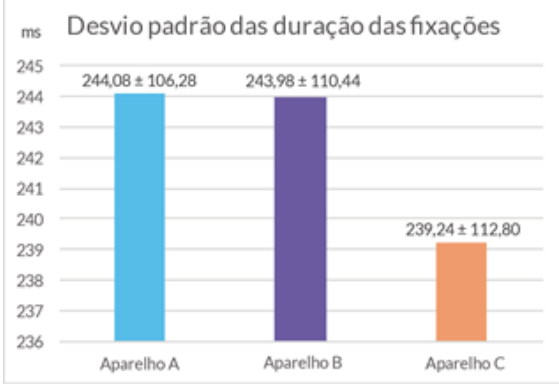

Figura 5- Gráfico duração das fixações

Já o contraste, segundo Khalighy et al. (2015), é uma relação entre pureza e proporção, sendo dependente do número de elementos e a proporção entre eles.

Nesse sentido, observa-se que $O$ aparelho $C$ obtém resultados positivos de acordo com os índices de beleza considerados, uma vez que atingiu menores valores de número de fixações e DP de duração das mesmas, consequentemente obtendo maiores valores de pureza, proporção e contraste. $\mathrm{O}$ aparelho $\mathrm{B}$ obteve maiores valores 
de contraste se comparado ao aparelho A, não obtendo diferenças significativas quanto à proporção.

A atração evocada por um estímulo é dependente de variáveis externas e pode evocar respostas emocionais, assim pode-se relacionar com as variações na dilatação da pupila (HO; LU, 2014). Apresentam-se os valores médios e desvio padrão para a dilatação da pupila na tarefa de olhar livre das imagens dos aparelhos A, B e C (Figura 6).

Verifica-se que o Aparelho $C$ obteve maiores valores médios de dilatação da pupila, podendo estar relacionado à atratividade do estímulo visual. Estudos anteriores observaram que tanto estímulos positivos quanto negativos resultam em maior dilatação da pupila quando comparada à estímulos neutros (BRADLEY et al. 2008). Enquanto em um segundo estudo (HO; LU, 2014), observou-se maior variação na dilatação da pupila quando da exposição à estímulos negativos, não apresentando variações significativas entre estímulos neutros e positivos, o estudo aponta ainda que a intensidade das emoções evocadas é menor quando o estímulo é um produto encontrando variações maiores para objetos que indicam perigo ou ameaça (LEBRECHT et al., 2012).

Constata-se que os modelos A e B não apresentaram diferenças significativas de dilatação da pupila, não possibilitando a hierarquização deste fator.

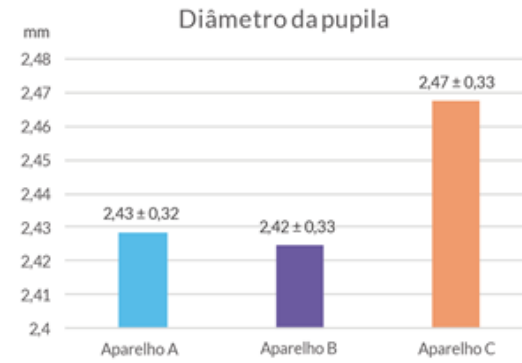

Figura 6- Gráfico diâmetro da pupila

Para elucidação da percepção de atração, além da dilatação da pupila leva-se em conta também os dados do questionário, apresentados em seguida, onde se considera atração uma relação entre familiaridade e novidade.

Na tarefa orientada, de verbalização da preferência, analisa-se tanto a preferência declarada, quanto os padrões visuais por meio dos mapas de calor. Observa-se uma maior preferência estética declarada pelo Aparelho C, seguido do modelo B (Figura 7), também nos mapas de calor nota-se a tendência de maior concentração do olhar no modelo preferido, indicado por meio da cor vermelha (Figura 8). No comparativo entre o modelo A e o modelo B (Figura 8), observa-se maior concentração do olhar no modelo B, já entre C e A (Figura 8) há maior centralização no modelo C. Assim como no comparativo entre B e C, com maior concentração em C (Figura 8).

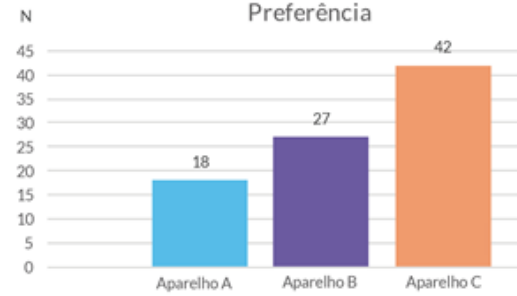

Figura 7- Gráfico preferência declarada

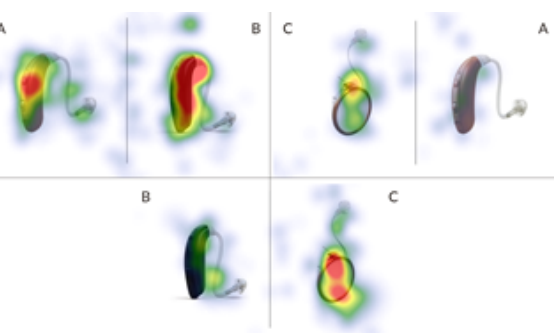

Figura 8- Mapas de calor

\subsection{Questionário}

Dos 29 sujeitos participantes da pesquisa, 72\% são do sexo feminino, nenhum usuário de aparelho auditivo ou com deficiência auditiva. Quanto a idade, 69\% dos participantes possuem idade entre 20 e 30 anos e também $69 \%$ tem ou já tiveram contato com usuário de aparelho auditivo (familiares, amigos ou conhecidos) (Figura 9).

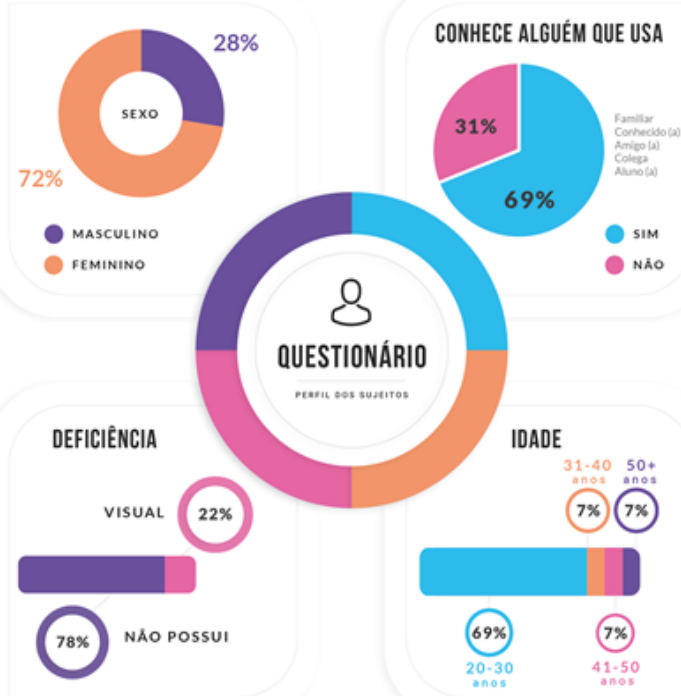

Figura 9- Infográfico dos dados obtidos do questionário

Verificando que $69 \%$ dos sujeitos tem ou já tiveram contato com usuários de aparelho auditivo, entende-se que a familiaridade com o dispositivo deve ser maior.

A partir da escala entre "familiar" e "novidade" avaliada pelos participantes para 
cada dispositivo, conforme apresentado nos métodos, o índice foi calculado entre -3 e 3. Assim, observa-se que o aparelho A tende a familiaridade, enquanto que o aparelho $B$ se aproxima do equilíbrio, o aparelho $C$ se aproxima da extremidade de novidade (Figura 10).

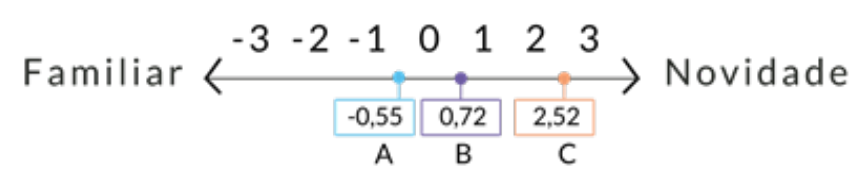

Figura 10- Escala de Familiaridade-novidade

Destaca-se que o nível de familiaridade ou novidade de um produto não está necessariamente associado a características positivas ou negativas, são fatores que influenciam na atratividade e dependem de demais variáveis.

Com base no rastreamento ocular da tarefa orientada, nota-se que o Aparelho $C$ se mostra um estímulo visual atraente, o que pode ser devido à sua não-peculiaridade de configurações estéticas, corroborando com a preferência declarada pelos usuários. O produto atendeu aos princípios de beleza definidos, no entanto a percepção do mesmo como inovador ao extremo poderia diminuir a atratividade estética, uma vez que o ser humano tende a buscar segurança nos estímulos reconhecidos (HEKKERT, 2014).

Ao observar a maior preferência declarada pelo aparelho na tarefa orientada em conjunto com a maior dilatação da pupila, entende-se que o modelo $\mathrm{C}$ se configurou como um estímulo visual atraente positivamente, confirmando a hipótese apresentada de acordo com os princípios estéticos de pureza, proporção e contraste. Já o aparelho A foi o que demonstrou pior desempenho quanto à percepção estética, não atendendo aos princípios de beleza mensurados por meio do rastreamento ocular, corroborando com a preferência declarada pelos sujeitos onde obteve a pior avaliação.

Compreende-se que os resultados podem estar relacionados ao estigma existente com relação ao aparelho auditivo comum (Aparelho A), enquanto o aparelho $C$, por possuir características estéticas diferenciadas, não possui significados prévios atribuídos pelos sujeitos, assim não sendo reconhecido como um símbolo de incapacidade. $O$ modelo de aparelho B obteve valores intermediários em pureza, proporção e contraste assim como na preferência declarada, possivelmente devido às suas configurações formais familiares que, entretanto, não despertam tão fortemente o estigma devido à utilização de cor.

Pontuam-se, portanto, algumas observações relativas ao uso do rastreamento ocular para avaliação da experiência estética de aparelhos auditivos:

- Os princípios de beleza, pureza, proporção e contraste apresentam aplicabilidade para a avaliação desses produtos;

- Como demonstrado na literatura (BRADLEY et al., 2008; HO; LU, 2014; PARTALA SURAKKA, 2003), a dilatação da pupila está relacionada à atratividade do estímulo ou a emoção derivada da experiência estética, que pode ser positiva ou negativa, devendo ser feita em conjunto com outra avaliação;

A experiência estética se dá por beleza (características do produto), e atração (influenciada por agentes externos), estando a última ligada a familiaridade do sujeito com o produto verificada por meio do questionário;
- O fator atração estética como o equilíbrio entre familiaridade e novidade não foi verificado no estudo, em que o modelo de aparelho descrito mais extremamente como inovador, foi também o preferido esteticamente;

- O mapa de calor do rastreamento ocular permitiu observar que o estímulo declarado como preferido esteticamente é também o que atrai o olhar em termos de número e tempo de fixações.

Com relação a estas contribuições, entende-se que ao mensurar a experiência estética de modo objetivo, pode-se compreender aspectos cognitivos e afetivos da interação com os produtos (GUO et al., 2016), o que em projetos de tecnologias assistivas pode auxiliar na evocação de emoções positivas atreladas a esses dispositivos. Sendo o rastreamento ocular uma medida fisiológica, está menos predisposta a alterações dos resultados devido à expressão de cada sujeito ou interferências do ambiente, caracterizando-se como um método de mensuração confiável se comparado à métodos subjetivos (ARIELY; BERNS, 2010).

Ainda, a utilização do rastreamento ocular para mensuração da experiência estética permite compreender a percepção da sociedade acerca daquele objeto, deixando de se basear apenas no entendimento do próprio projetista e reforçando a centralidade do ser humano no desenvolvimento do projeto.

\section{Considerações Finais}

A interação entre usuário e produto depende de diversos fatores, tanto das necessidades e expectativas do usuário, como das características do produto. Essa interação pode inclusive influenciar na formação da identidade do usuário. Em se tratando de Tecnologias Assistivas, como o aparelho auditivo, os valores simbólicos atrelados às características do produto tendem a ser associados com significados negativos, que afetam autoestima e, consequentemente, a qualidade de vida dos usuários.

Considerando a problemática tratada nesta pesquisa, ao compreender as percepções do ser humano quanto ao aparelho auditivo, espera-se contribuir para a redução do estigma e de comportamentos discriminatórios ou exclusivos para com os usuário dessa Tecnologia Assistiva. Nesse sentido, destaca-se também a importância de inclusão do design no projeto de TA para compreensão do ser humano como um todo e igualmente considerar as dimensões prática, estética e simbólica do produto. No que diz respeito ao objetivo da pesquisa, de mensuração da percepção estética visual de 3 diferentes modelos de aparelhos auditivos, tem-se relações dos padrões visuais identificados por meio do rastreamento com alguns princípios de estética: foi possível relacionar o desvio padrão de duração das fixações com a proporção da forma, assim como o número de fixações com a pureza, consequentemente possibilitando a avaliação do contraste. Além disso, a mensuração do diâmetro da pupila aliado ao nível de familiaridade ou novidade declarado pelos sujeitos, permitiu avaliar a atratividade dos estímulos. Esses resultados corroboraram com a preferência declarada pelos usuários, que por sua vez foi demonstrada também pelo mapa de calor, indicando que a fixação visual é maior nos estímulos preferidos. Já a avaliação entre familiaridade e novidade verificou que o modelo preferido atingiu a pontuação mais extrema em termos de novidade, não se identificando a relação de equilíbrio descrita na literatura. 
O que pode estar relacionado com fatores específicos do estigma existente para com esses produtos.

Ainda, observa-se que o rastreamento ocular é um método que oferece a possibilidade de quantificação da percepção visual assim atribuindo objetividade ao processo, podendo ser utilizado para adequação das características estéticas do aparelho auditivo às preferências do usuário. Além disso, a compreensão das relações entre as características estéticas do objeto e percepção do sujeito, pode guiar o desenvolvimento de aparelhos auditivos atrelados à significados positivos, tanto para o usuário quanto para a sociedade.

Dessa forma, destaca-se a oportunidade de identificar em estudos futuros como se dá a relação dos fatores de agradabilidade e atratividade estética com o estigma relacionado às tecnologias assistivas.

\section{REFERÊNCIAS}

ARIELY, Dan; BERNS, Gregory S. Neuromarketing: the hope and hype of neuroimaging in business. Nature reviews neuroscience, v. 11, n. 4, p. 284, 2010.

BERGHMAN, M.; HEKKERT, P. Towards a unified model of aesthetic pleasure in design. New Ideas in Psychology, v. 47, p. 136-144, 1 dez. 2017.

BRADLEY, Margaret M. et al. The pupil as a measure of emotional arousal and autonomic activation. Psychophysiology, v. 45, n. 4, p. 602-607, 2008.

COATES, Del. Watches tell more than time: Product design, information, and the quest for elegance. London: McGraw-Hill, 2003.

CRILLY, N.; MOULTRIE, J.; CLARKSON, P. J. Seeing things: Consumer response to the visual domain in product design. Design Studies, v. 25, n. 6, p. 547-577, 2004.

CUNHA, Julia Marina; MERINO, Giselle. Design e Tecnologia Assistiva: O aparelho auditivo como acessório de moda. In: MEDOLA, Fausto Orsi; PASCHOARELLI, Luis Carlos (Org.) Tecnologia Assistiva: Pesquisa e Conhecimento. Bauru: Canal 6, 2018. p. 391-400.

DIEFENBACH, Sarah; HASSENZAHL, Marc. The dilemma of the hedonic-Appreciated, but hard to justify. Interacting with Computers, v. 23, n. 5, p. 461-472, 2011.

DOUCHOVA, V.; NESETRIL, J. Fragment of Aesthetic Processing. WDS'09 Proceedings of Contributed Papers, p. 7-10, 2009.

GOBBI, Aline Girardi et al. Uso Do Eye Tracking Para Obtenção De Medidas Quantitativas Em Testes De Usabilidade: Um Estudo Focado Na Medida Da Satisfação. Human Factors In Design, [s.I.], v. 6, n. 11, p.106-125, 13 dez. 2017. Universidade do Estado de Santa Catarina.

GOFFMAN, Erving. Stigma and social identity. In: Deviance and Liberty. Routledge, 2018. p. 24-31.
GRANKA, Laura; FEUSNER, Matthew; LORIGO, Lori. Eye monitoring in online search. In: Passive eye monitoring. Springer, Berlin, Heidelberg, 2008. p. 347-372.

GUO, F. et al. Can eye-tracking data be measured to assess product design?: Visual attention mechanism should be considered. International Journal of Industrial Ergonomics, v. 53, p. 229-235, 2016.

HEKKERT, P. Aesthetic responses to design: a battle of impulses. In: TINIO, P. P. L.; SMITH, J. K. (Eds.). The Cambridge Handbook of the Psychology of Aesthetics and the Arts. Cambridge: Cambridge University Press, 2014. v. xip. 277-299.

HO, C. H.; LU, Y. N. Can pupil size be measured to assess design products? International Journal of Industrial Ergonomics, v. 44, n. 3, p. 436-441, 1 maio 2014.

HSIAO, S. W.; CHIU, F. Y.; CHEN, C. S. Applying aesthetics measurement to product design. International Journal of Industrial Ergonomics, v. 38, n. 11-12, p. 910-920, 1 nov. 2008.

JACOBSON, Susanne. Managing Stigma and Expressing the Self. 2014. 332 f. Tese (Doutorado) - Curso de Design And Architecture, Aalto University, Helsinki, 2014.

KATZ, Helena. Por uma teoria crítica do corpo. In: OLIVEIRA, Ana Claudia de; CASTILHO, Kathia (Org.). Corpo e moda: por uma compreensão do contemporâneo. Barueri, Sp: Estação das Letras e Cores, 2008. p. 69-74.

KHALIGHY, S. et al. Measuring aesthetic in design. Proceedings of International Design Conference, v. 2014-Janua, p. 2083-2094, 2014.

KHALIGHY, S. et al. Quantifying the qualities of aesthetics in product design using eyetracking technology. International Journal of Industrial Ergonomics, v. 49, p. 31-43, 2015.

LEBRECHT, Sophie et al. Micro-valences: perceiving affective valence in everyday objects. Frontiers in psychology, v. 3, p. 107, 2012.

LÖBACH, Bernd. Design industrial: bases para a configuração dos produtos industriais. Edgard Blücher, 2001.

MOSHAGEN, M.; THIELSCH, M. T. Facets of visual aesthetics. International Journal of Human Computer Studies, v. 68, n. 10, p. 689-709, 2010.

NAYAK, B. K.; KARMAKAR, S. Eye Tracking Based Objective Evaluation of Visual Aesthetics: A Review. (F. Rebelo, M. M. Soares, Eds.)Advances in Ergonomics in Design. Anais...Cham: Springer International Publishing, 2019.

NORMAN, Donald A. Design emocional: por que adoramos (ou detestamos) os objetos do dia-a-dia. Rocco, 2008. 
PAPE, T. L. B.; KIM, J.; WEINER, B. The shaping of individual meanings assigned to assistive technology: A review of personal factors. Disability and Rehabilitation, v. 24, $\mathrm{n}$. 1-3, p. 5-20, 2002.

PARTALA, Timo; SURAKKA, Veikko. Pupil size variation as an indication of affective processing. International Journal Of Human-computer Studies, [s.I.], v. 59, n. 1-2, p.185198, jul. 2003. Elsevier BV. http://dx.doi.org/10.1016/s1071-5819(03)00017-x.

PATRICK, V. M. Everyday consumer aesthetics. Current Opinion in Psychology, v. 10, p. 60-64, 2016.

PHAM, Binh. Design for aesthetics: interactions of design variables and aesthetic properties. In: Human Vision and Electronic Imaging IV. International Society for Optics and Photonics, 1999. p. 364-372.

POPELKA, G. R. et al. Hearing Aids. Cham: Springer International Publishing, 2016. v. 56

PRODANOV, Cleber Cristiano; DE FREITAS, Ernani Cesar. Metodologia do trabalho científico: métodos e técnicas da pesquisa e do trabalho acadêmico- $2^{a}$ Edição. Editora Feevale, 2013.

PULLIN, Graham. Design meets disability. MIT press, 2009.

SHINOHARA, K.; WOBBROCK, J. O. In the shadow of misperception: Assistive technology use and social interactions. Conference on Human Factors in Computing Systems Proceedings. Anais...2011

\section{AGRADECIMENTOS}

As autoras agradecem à CAPES, ao Núcleo de Gestão de Design e Laboratório de Design e Usabilidade da Universidade Federal de Santa Catarina (NGD/LDU), e à Rede de Pesquisa e Desenvolvimento em Tecnologia Assistiva (RPDTA) por viabilizarem essa pesquisa.

O presente trabalho foi realizado com apoio da Coordenação de Aperfeiçoamento de Pessoal de Nível Superior - Brasil (CAPES) - Código de Financiamento 001 